\title{
Inductive measurement and encoding of k-space trajectories in MR raw data
}

\author{
Pedersen, Jan Ole; Hanson, Christian G.; Xue, Rong; Hanson, Lars G.
}

Published in:

Magnetic Resonance Materials in Physics, Biology and Medicine

Link to article, DOI:

10.1007/s10334-019-00770-2

Publication date:

2019

Document Version

Peer reviewed version

Link back to DTU Orbit

Citation $(A P A)$ :

Pedersen, J. O., Hanson, C. G., Xue, R., \& Hanson, L. G. (2019). Inductive measurement and encoding of kspace trajectories in MR raw data. Magnetic Resonance Materials in Physics, Biology and Medicine, 32(6), 655-667. https://doi.org/10.1007/s10334-019-00770-2

\section{General rights}

Copyright and moral rights for the publications made accessible in the public portal are retained by the authors and/or other copyright owners and it is a condition of accessing publications that users recognise and abide by the legal requirements associated with these rights.

- Users may download and print one copy of any publication from the public portal for the purpose of private study or research.

- You may not further distribute the material or use it for any profit-making activity or commercial gain

- You may freely distribute the URL identifying the publication in the public portal 
Noname manuscript No.

(will be inserted by the editor)

\section{Inductive measurement and encoding of $k$-space trajectories in MR raw data}

Jan Ole Pedersen ${ }^{1,2,3}$ • Christian G. Hanson •

Rong Xue $\mathrm{X}^{4,5}$ - Lars G. Hanson ${ }^{1,2, *}$

Accepted for Magnetic Resonance Materials in Physics, Biology and Medicine, July 2019

1 Abstract

Objectives Concurrently acquire an inductively generated $k$-space trajectory measure and magnetic resonance imaging data by an MR scanner.

Materials and methods Inductive 1D gradient measures were regularized using measured gradient coil currents and recorded individually by the scanner concurrently with raw MR data. Gradient measures were frequency modulated into an RF signal receivable by the scanner, yielding a $k$-space trajectory measure from the cumulat-

\footnotetext{
${ }^{1}$ Center for Magnetic Resonance, Department of Electrical Engineering, Technical University of Denmark, Kgs. Lyngby, Denmark

2 Danish Research Centre for Magnetic Resonance, Centre for Functional and Diagnostic Imaging and Research, Copenhagen University Hospital, Hvidovre, Denmark

${ }^{3}$ Sino-Danish Center for Education and Research, Aarhus, Denmark

4 State Key Laboratory of Brain and Cognitive Science, Institute of Biophysics, Chinese Academy of Sciences, Beijing, China

${ }^{5}$ Sino-Danish College, University of Chinese Academy of Sciences, Beijing, China

* Correspondence to:lghan@dtu.dk
} 
ive phase of the acquired data. Generation of the gradient measure and frequency modulation was performed by previously developed custom, versatile circuitry.

Results For a normal echo planar imaging (EPI) sequence, the acquired $k$-space trajectory measure yielded slightly improved image quality compared to that obtained from using the scanner's estimated eddy current compensated $k$-space trajectory. For a spiral trajectory, the regularized inductive $k$-space trajectory measure lead to a $76 \%$ decrease in the root-mean-square error of the reconstructed image.

Discussion While the proof-of-concept experiments show potential for further improvement, the feasibility of inductively measuring $k$-space trajectories and increasing the precision through regularization was demonstrated. The approach may offer an inexpensive method to acquire $k$-space trajectories concurrently with scanning.

\section{Introduction}

Real-time acquisition and processing of non-MR signals in the MR environment is of general interest. A particular example is monitoring of the temporally varying gradient field during $\mathrm{MR}$ image acquisition, and thereby of actual $k$-space trajectories, which may differ significantly from nominal trajectories $[1,2,3]$. The deviations are caused by gradient imperfections that are normally addressed by the use of robust $k$-space trajectories where hardware limitations and instabilities cause limited, or easily accounted for, artefacts in reconstructed images. One example is echo planar imaging (EPI), where Nyquist ghosting, caused by deviation from the nominal $k$-space trajectory in the readout-direction, typically is 
addressed by acquiring extra reference lines to determine and facilitate correction of an effective delay in the play-out of the gradient waveforms [4].

Other $k$-space trajectories are more challenging, e.g., spiral or rosette trajectories, as the effect of gradient imperfections are not as easily accounted for. For such sequences, nominal $k$-space trajectories are typically not sufficient, and $k$ space trajectory errors must either be alleviated or corrected for in reconstruction using more elaborate techniques [5]. One approach is to assume time invariant behaviour of the gradient system, which allows for determining the $k$-space trajectory from calibration scans $[6,7,8]$ or convolving nominal trajectories with predetermined impulse-response functions to improve the estimate of the actual gradient waveforms $[9,10,11]$. Particularly, the method of Duyn et al. [6] is widely applied as it yields reliable $k$-space trajectory measures in reasonable time, while only requiring the use of a homogeneous phantom and moderate sequence alterations. The assumption of time invariance is challenged by temporal changes in the gradient system induced by, e.g., heating of the gradient coils due to ohmic losses during gradient-intensive sequences. Hardware temperatures and associated imperfections typically change over seconds to minutes, whereas other characteristics may change on a longer time scale, e.g., when the system is recalibrated or serviced. Additionally, unavoidable minor shot-to-shot variability happen on an even shorter time scale due to hardware instabilities [3]. NMR-based field probes offer attractive ways to determine magnetic fields dynamically, and therefore also $k$-space trajectories concurrently with imaging $[1,3,12,13]$, avoiding the assumption of time invariance. While offering excellent sensitivity, they require extensive dedicated hardware specific to scanners and field strength. They may interfere with imaging [14], even when based on nuclei other than the one being imaged, as 
they rely on diamagnetic and conductive materials that may perturb the $B_{0}$ and

RF fields. This is particularly challenging as they are highly sensitive to magnetic field inhomogeneity, and therefore must be placed inside or close to the field of view (FOV).

The time varying property of the gradient field allows for alternative direct monitoring of the gradient performance. While field probes offer better sensitivity, inductive measurements do not rely on the acquisition of an MR signal (FID), thus offering more robustness against field inhomogeneity and independence of relaxation and spin history. The inductive signal is proportional to the time-derivative of the magnetic field, and obtaining a measure for the gradient field therefore requires temporal integration. In the work of Senaj et al. [15], this integration was performed in analogue circuitry that in addition featured a relay for discharging of the integrator at the start of each measurement. The integration necessary to determine the gradient field inductively can alternatively be performed digitally, potentially simplifying the necessary hardware and yielding increased flexibility in signal processing capabilities [16].

Estimating $k$-space trajectories from measured gradients involves an additional temporal integration, and doing this concurrently with scanning requires real-time signal processing and sub- $\mu$ s synchronization with the MR signal acquisition. Particularly the synchronization is non-trivial to obtain, but can be achieved directly if the scanner itself is used for acquisition of both MR and non-MR signals [17], which we recently developed open-source circuitry to facilitate $[18,19]$. This features a field-programmable gate array (FPGA) for fast and re-programmable signal processing, and acquisition of up to 4 input signals with one input being designated for acquisition of RF power correlates to determine the envelope of scanner-applied 
RF pulses. The FPGA controls the frequency and amplitude of a direct digital synthesis sub-circuitry (DDS) that can generate signals receivable by typical MR scanners.

In this study, gradient-induced magnetic field changes of an MR scanner are measured inductively, and used for generating 1D $k$-space trajectory measures through digital integration and real-time processing by the previously developed custom circuitry. Performing frequency modulation of a carrier signal with an integrated gradient measure encodes the $k$-space trajectory into the cumulative phase of the signal. If the frequency of the carrier signal is in the range of Larmor frequencies of the imaged sample, it can be transmitted via the receive chain of the scanner without being attenuated by its filters. The demodulation performed in the receive chain undoes the frequency modulation, enabling extraction of a $k$-space trajectory measure from raw data acquired concurrently with MR data. The inherent integrations needed for determining $k$-space trajectories inductively cause noise to accumulate rapidly over time, which decreases the obtainable precision. We show that the noise accumulation can be counteracted by regularization using simple measurements of the current running to the gradient coils, i.e. stabilizing the signal by limiting noise amplification. The $k$-space trajectory caused by the readout gradient of an EPI sequence, and the $k$-space trajectory of a spiral sequence are measured, and used in reconstruction of MR data from a structured water phantom. The reproducibility, accuracy and ability to reconstruct artefactfree images are evaluated for the prototype implementation to assess the general applicability of the method for reliably determining $k$-space trajectories concurrently with MR acquisition. 


\section{Theory}

The gradient coils of a scanner generate a field $\mathbf{B}_{g}(\mathbf{r}, t)$ varying in direction and strength over space and time. For the sake of Fourier imaging, this field is within the imaging volume ideally directed along the static field (unit vector $\hat{\mathbf{z}}$ ) and is linearly varying along the intended direction specified by a gradient vector $\mathbf{G}(t)$. The actual field deviates by an amount $\mathbf{B}_{g}^{\prime}(\mathbf{r}, t)$ due to unavoidable spatial nonlinearities and concomitant fields that are unwanted components orthogonal to the main field.

$$
\mathbf{B}(\mathbf{r}, t)=\mathbf{B}_{0}(\mathbf{r})+(\mathbf{G}(t) \cdot \mathbf{r}) \hat{\mathbf{z}}+\mathbf{B}_{g}^{\prime}(\mathbf{r}, t)
$$

Due to eddy currents and amplifier limitations, the temporal field variation generally deviates from the ideal variation often visualized in sequence diagrams. Various spatial components (e.g. local fields) may have differing temporal dependence, but to a typically good approximation, the field induced by eddy currents is spatially independent or amounts to temporal gradient strength distortions that can be characterized by temporal impulse response functions describing the field variation resulting from ramping of gradients $[10,11,13,20]$. The temporal impulse response may itself vary, depending on system temperature, for example [3]. In the present communication demonstrating proof of concept, we focus on the temporal field imperfections only, and use a simplified model of the field. We adopt the normal ideal model consistent with Fourier imaging and the notion of $k$-space trajectories common to all nuclei within the imaged sample, i.e. $\mathbf{B}_{g}^{\prime}(\mathbf{r}, t)$ is ignored. The presented methods therefore do not address effects of non-linearities, concomitant fields, or other ignored field components. 
The $\hat{\mathbf{x}}, \hat{\mathbf{y}}$ and $\hat{\mathbf{z}}$ components of $\mathbf{G}(t)$ are normally generated by three independent sets of coils and amplifiers. We focus the theory section on any of these components individually and choose $G=G_{x}, G_{y}$ or $G_{z}$ with the other two components being zero. Following excitation, the position, $k(t)$, in the corresponding one-dimensional $k$-space at time $t$ due to the time-dependent, spatially linear gradient field, $G(t)$, is given as

$$
k(t)=\gamma \int_{0}^{t} G(\tau) d \tau
$$

where $t=0$ is the isodelay point [21] of the excitation pulse, and $\gamma$ is the gyromagnetic ratio $\left(42.58 \mathrm{MHz}^{-1}\right.$ for protons). The $k$-space trajectory can then be determined from the accumulated phase, $\phi(t)=\int_{0}^{t} \omega(\tau) d \tau$, of a signal originating at position $\mathbf{r}$, with angular frequency $\omega(t)=2 \pi \gamma D G(t)$ with $D$ being the component of $\mathbf{r}$ along the gradient direction.

Inductive measurement of all temporally varying components of the field is in principle possible with a sufficiently dense array of independent pick-up coils oriented to be sensitive to the field components of interest. According to Faraday's law, the induced voltage $V(t)$ over a stationary small circular pick-up coil characterized by a magnetic dipole sensitivity $\mathbf{d}$ orthogonal to the wire loop, is proportional to the field change along $\mathbf{d}$ :

$$
V(t) \propto \frac{d}{d t}\left(\mathbf{d} \cdot \mathbf{B}\left(\mathbf{r}_{0}, t\right)\right)
$$

where $\mathbf{r}_{0}$ is the position of the pick-up coil. Choosing the loop surface normal along $\hat{\mathbf{z}}$, the voltage $V(t)$ induced by a temporally varying magnetic flux, $\Phi(t)$, caused by the gradient $G(t)$ is:

$$
V(t)=-\frac{d \Phi(t)}{d t}=\frac{d G(t)}{d t} \frac{1}{b} .
$$


The constant $b$ is dependent on the geometry of the pick-up coil and its position in the gradient coil of the MR scanner. Under the assumption that $G(t)$ is slowly varying compared to the dwell time $(\Delta t)$ between discrete samples of $V(t)$, it can be well approximated by numerical integration, e.g., by simple updating of an inductive gradient estimate $G^{V}(t)$ :

$$
\begin{aligned}
& G^{V}(t)=G^{V}(t-\Delta t)+b V(t) \Delta t, \quad \text { or equivalently } \\
& G^{V}(t)=\sum_{i=1}^{N}\left[b V\left(t_{1}+i \Delta t\right) \Delta t\right]+c=G(t)+\epsilon(t) .
\end{aligned}
$$

Here $N=\frac{t-t_{1}}{\Delta t}$, with $t_{1}$ being the starting time of integration. The constant $c$ accounts for any gradient applied at $t_{1}$, and $\epsilon(t)$ accounts for any deviation between the gradient estimate and the actual gradient, $G(t)$. Due to the integration (approximated by summation) involved in determining $G^{V}(t)$, the error $\epsilon(t)$ is expected to increase in amplitude over time, limiting the obtainable sensitivity. More specifically, a sampling bias, e.g. due to round-off, will add a linearly increasing bias to the gradient estimate, and the variance due to the accumulated error $\epsilon(t)$ will increase linearly with time, if sampling errors are normally distributed with zero mean, uncorrelated, and have constant variance over time.

Noise amplification upon further integration to calculate $k(t)$ can be counteracted by regularization (signal stabilization) based on additional knowledge of $G(t)$. One possibility is to calculate a gradient estimate, $G^{I}(t)$, from its driving coil current, $I(t)[22]$. This is an approximate gradient field measure insensitive to, e.g., eddy currents, and an inaccurate predictor over short time scales. However, on a longer time scale it is highly reproducible and relatively accurate, and therefore potentially well suited for regularization of the inductive magnetic field measure, i.e. avoidance of overfitting to noise. A simple, regularized inductive measure, $G^{\mathrm{reg}}(t)$, 
can be calculated using a modified updating rule (replacing Eq. 5) for the gradient estimate

$$
\begin{aligned}
& G^{\mathrm{reg}}(t)=G^{\mathrm{reg}}(t-\Delta t)+b V(t) \Delta t-\lambda R(t-\Delta t), \quad \text { or equivalently } \\
& G^{\mathrm{reg}}(t)=\sum_{i=1}^{N}\left[b V\left(t_{1}+i \Delta t\right) \Delta t-\lambda R\left(t_{1}+(i-1) \Delta t\right)\right]+c,
\end{aligned}
$$

The non-negative $\lambda$ determines the influence of the regularization term

$$
R(t)=\zeta \cdot\left[G^{\mathrm{reg}}(t)-G^{I}(t)\right]^{2}
$$

If $G^{\mathrm{reg}}(t)<G^{I}(t)$, then $\zeta=-1$ for the given sample of $R(t)$, else, $\zeta=1$. The term $R(t)$ will drive the regularized induction gradient measure, $G^{\mathrm{reg}}(t)$, towards the current-based estimate, $G^{I}(t)$, at a rate dependent on $\lambda$. The driving term is chosen to be quadratic in the discrepancy between the two measures, so small excursions from $G^{I}(t)$ are permitted whereas larger deviations will soon be corrected. The delay by $\Delta t$ of the regularization term in Eq. 7 is a necessity due to hardware constraints described later. Assuming that the amplitude of the gradient estimate error, $\epsilon(t)$, is slowly increasing compared to the rate of change of $G(t)$, for an appropriate chosen value of $\lambda, G^{\text {reg }}(t)$ retains the accuracy from an inductive measure of $G(t)$ on a short time scale, while having reproducibility similar to $G^{I}(t)$ on a longer time scale.

If a gradient estimate is used to frequency-modulate (FM) the carrier of an $\mathrm{RF}$ transmitter oscillating within the detection range of a scanner's RF receiver, the unwrapped phase of the signal recorded by the scanner will reflect the $k$-space position, according to Eq. 2. If the scaling of the frequency modulation is chosen to make the transmitter oscillate at the frequency that a spin isochromate at distance $D$ from the isocenter along the gradient direction would have, except for an offset 
$\Delta \omega$, then the unwrapped phase becomes

$$
\phi(t)=2 \pi D k(t)+\Delta \omega t+\phi_{1} .
$$

$\Delta \omega$ is the offset between the unmodulated FM carrier $(G=0)$ and the demodulation frequency of the scanner. Phase unwrapping is facilitated by the two being near equal and by high SNR that can also make scanner sample noise insignificant. $\phi_{1}$ is the initial phase when the integration starts at time $t_{1}$. The $k$-space trajectory can be calculated as $k(t)=\phi(t) /(2 \pi D)$ if $\phi_{1}$ and the linear term is subtracted from the phase beforehand, e.g. estimated from a linear fit of the recorded phase in the absence of gradient. Clock-jitter and discrete modulator frequency updating will introduce additional noise on the $k(t)$-estimate, but this does not accumulate, and is expected to simply increase the noise on $k(t)$.

Whereas the gradient estimate noise variance is expected to increase approximately linearly with time after excitation as described above, the $k$-space estimate noise variance will increase with time to the third power under the same assumptions [23], unless regularization is done. This is due to the extra noiseamplifying integration. Since regularization keeps the gradient estimate near the current-based estimate, $G^{I}(t)$, the noise variance of the regularized $k(t)$-estimate is expected to asymptotically increase linearly with time under an assumption of random zero-mean current sample noise. A biased current measure, e.g. from systematic round-off error, will bias $G(t)$, and therefore the carrier frequency, but the above-mentioned fit aimed at removing a carrier offset will also remove any such bias. 
Tab. 1 Summary of acquired and compared $k$-space trajectory measures. When appropriate, an additional subscript $\{s, m, x, y\}$ denotes the played out $k$-space trajectory component, where $m$ denotes EPI readout direction, and $x, y$ the two components of a spiral $k$-space trajectory vector, combined denoted $s$.

\begin{tabular}{ll}
\hline$k$-space trajectory & Obtained from... \\
\hline$k^{\text {scan }}(t)$ & $\ldots$ scanner logfile (estimated). \\
$\tilde{k}^{\text {scan }}(t)$ & $\ldots k^{\text {scan }}(t)$ by correcting for an effective delay due to eddy currents. \\
$k^{V}(t)$ & $\ldots$ inductively measuring the gradient field. \\
$k^{I}(t)$ & $\ldots$ a measure of the current delivered to the gradient coil. \\
$k^{\text {reg }}(t)$ & $\ldots$ regularizing an inductive measure by a current measure. \\
$k^{\text {Duyn }(t)}$ & $\ldots$ Duyn's method, relying on 1D imaging after localized excitation. \\
$k_{0}(t)$ & $\ldots$ determining $k(t)$ without applying gradients (ideally zero).
\end{tabular}

\section{Materials and methods}

Multiple $k$-space trajectory measures were determined and compared. These are introduced in the following section and summarized in Tab. 1.

To determine $V(t)$, a simple pick-up coil (20 windings, $\varnothing 3 \mathrm{~cm}$ ) was fixed to a head coil of the MR scanner $(7 / 15 \mathrm{~cm}$ in $\hat{\mathbf{x}} / \hat{\mathbf{y}}$-direction from isocenter, with the $\hat{\mathbf{y}}$-direction being posterior-anterior for normal suppine positioning), and with its surface perpendicular to the $\hat{\mathbf{z}}$-direction. Literature suggests mechanical vibration of the patient table being a potential challenge [24], and prior electroencephalography experiments using the applied MR scanner indicated this being a significant noise source. A blanket was therefore placed between the patient table and the 
head coil to reduce vibrations of the pick-up coil. Rather than aiming at highly accurate positioning to absolutely quantify gradients from voltage measures, amplitudes were instead calibrated by estimating the scaling needed to give the correct spatial dimensions after image reconstruction. If the alignment of the pick-up coil is not perfect, it will be slightly sensitive to concomitant fields, but these have the same temporal dependence as the imaging gradients, except for minor eddy current contributions that may differ between directions. Hence, the calibration also compensates detrimental effects of potential misalignment of the pick-up coil. Since it records flux changes and does not move in the static field (except possibly by vibration), it is relatively insensitive to varying fields not caused by gradients, e.g., low-frequency field changes induced by movement of the largely diamagnetic tissue of a patient.

A current clamp was used to determine $I(t)$ (Fluke i400s, Fluke Corporation, Everett, WA, USA. Current range: $5 \mathrm{~A}$ to $400 \mathrm{~A})$. The pick-up coil and the current clamp were connected to the above-mentioned previously developed circuitry facilitating sampling of non-MR signals by MR scanners [18]. This was achieved through real-time FPGA signal processing and modulation of a carrier signal recordable by the scanner. The circuitry determined $G^{V}(t), G^{I}(t)$, and $G^{\mathrm{reg}}(t)$ from discrete sampling of $V(t)$ and $I(t)$ (16-bit samples acquired at $200 \mathrm{kHz}$ sampling rate, $22 \mathrm{kHz}$ cutoff frequency anti-aliasing filter). A sub-bit bias of the ADCs of the circuitry introduced systematic errors in the measures of $V(t)$ and $I(t)$ causing linearly increasing biases in $G^{V}(t)$ and $k^{I}(t)$, respectively. These were removed by nulling of the least significant bit in the discretized $V(t)$ and $I(t)$ in a fraction of the time steps, which is a standard method of dealing with systematic digitization errors. The frequency of nulling was chosen, so that the period between $k$-space 
zero crossings matched those of the nominal trajectory on average. Circuitrytesting did not reveal problematic RF emission, but to facilitate use, including monitoring of FPGA-internal signals on a computer, the circuitry was positioned outside the scanner's Faraday cage. A carrier signal with frequency chosen equal to the scanner's reference frequency, $\omega_{0}$, was frequency modulated by the individual measures of $G(t)$ to yield $\omega^{\prime}(t)$. This signal was transmitted by wire to a single receive channel of the scanner. The demodulation performed by the scanner undid the frequency modulation except for inaccuracy $\Delta \omega$. As described in the theory section, this allowed for extraction of the individual measures of $k(t)$ from unwrapping of the cumulative phase of the scanner-acquired signal. The average of samples acquired during the first $50 \mu$ s of each $k$-space trajectory acquisition was subtracted to account for the initial phase $\phi_{1}$. Using a $3 \mathrm{~T}$ Philips Achieva scanner (software release 3.2 .3 ), the $k$-space trajectory due to gradients in only the readout-direction, $k_{m}(t)$, of a vendor EPI sequence was determined (TE: $35 \mathrm{ms,}$ TR: $4000 \mathrm{~ms}$, single slice, $2 \mathrm{~mm}$ thickness, FOV: $230 \times 230 \mathrm{~mm}, 96 \times 95$ acquisition matrix with $2 \times$ oversampling in the readout-direction yielding an actual acquisition matrix of $192 \times 95$, dwell time/echo-spacing: 4.6/493 $\mu \mathrm{s}, 50$ repetitions, $90^{\circ}$ excitation pulses). As summarized in Tab. 1, the trajectory was determined using $G_{m}^{V}(t)$ exclusively, yielding $k_{m}^{V}(t)$, using $G_{m}^{I}(t)$ exclusively $\left(k_{m}^{I}(t)\right)$, and using $G_{m}^{\text {reg }}(t)\left(k_{m}^{\text {reg }}(t)\right)$. In addition, Duyn's method was used to determine the $k$-space trajectory $\left(k_{m}^{\text {Duyn }}(t)\right)$ with three slice positions in the y-direction $(30 \mathrm{~mm},-30 \mathrm{~mm}$, $0 \mathrm{~mm})$. The acquisition of $G_{m}^{V}(t)$ was performed simultaneously with the slice offset of $30 \mathrm{~mm}, G_{m}^{I}(t)$ simultaneously with a slice offset of $-30 \mathrm{~mm}$, and $G_{m}^{\mathrm{reg}}(t)$ without a slice offset. The readout gradient was applied in the $\hat{\mathbf{x}}$-direction, corresponding to left-right in the scanner, so $G_{m}(t)=G_{x}(t)$. A correlate of the amplitude of RF 
pulses transmitted by the scanner was measured wirelessly by the applied circuitry using a quarter wavelength antenna placed inside the scanner's Faraday cage (position not critical). The approximate isodelay points of the excitation pulses were determined by this signal exceeding a threshold. A temporal gap between each excitation pulse and the start of $G_{m}(t)$ allowed for setting the estimate of $G_{m}(t)$ to 0 following each detection of an excitation pulse. This lead to $t_{1}$ being a time point without gradient activity, so $c=V\left(t_{1}\right)=0$ in Eq. 6 and Eq. 8. The long TR of $4000 \mathrm{~ms}$ was chosen to ensure minimal signal loss from incomplete MR signal relaxation, and to avoid heating effects of the gradient coils to ensure minimal variance over time, as needed for acquiring trajectories using Duyn's method as reference. To assess the circuitry performance and disentangle noise sources and biases, the unmodulated carrier signal was acquired in a separate scan $\left(k_{0}(t)\right)$, corresponding to determining $k(t)$ for $G(t)=0$.

The regularized, inductively determined $k_{m}^{\mathrm{reg}}(t)$ was interpolated to earlier time points to account for a delay $(15.1 \mu \mathrm{s})$ arising from passing signals through the circuitry, and from performing signal processing. The delay was determined using methodology similar to that presented by Bruder et al. [4]. The acquired $k_{m}^{I}(t)$ was re-sampled to account for an observed pre-emphasis in the current generating the magnetic field, and a delay originating from generating $G^{I}(t)(11.0 \mu \mathrm{s})$. The linear phase from $\Delta \omega$ being non-zero with a potential contribution from biased current measurements, was accounted for by subtracting a linear fit to the unwrapped phase of individual repetitions of the scans. The $k$-space trajectory estimated by the scanner, $k_{m}^{\mathrm{scan}}(t)$, was obtained from logging done by the scanner. This trajectory was re-sampled to shifted time points, yielding $\tilde{k}_{m}^{\text {scan }}(t)$, to account for an 
observed effective delay compared to $k_{m}^{\text {Duyn }}(t)(1.4 \mu \mathrm{s})$. This is similar to simple eddy current compensation.

EPI of a structured water phantom was performed with both readout and phase-encoding gradients. Imperfections in the blipped phase-encoding gradients were not limiting for the EPI performance, and were ignored in the present context. The acquired data were therefore reconstructed using the individual measures of $k_{m}(t)$ and nominal phase-encoding gradients $\left(G_{y}(t)\right)$, to yield $2 \mathrm{D}$ images.

In addition to the EPI sequence, the $k$-space trajectory of a single-shot spiralout sequence was also determined (TE: $10 \mathrm{~ms}$, TR: $4000 \mathrm{~ms}$, single slice, $2 \mathrm{~mm}$ thickness, FOV: $230 \times 230 \mathrm{~mm}, 9099$ samples, dwell time: $4.4 \mu \mathrm{s}, 50$ repetitions, $90^{\circ}$ excitation pulses). The spiral trajectory, $\mathbf{k}_{s}(t)$ was obtained from separate acquisitions of $k_{x}(t)$ and $k_{y}(t)$ using the regularized integrative measure $\left(\mathbf{k}_{s}^{\mathrm{reg}}(t)\right)$, and Duyn's method $\left(\mathbf{k}_{s}^{\text {Duyn }}(t)\right)$. Also the scanner's assumed trajectory $\left(\mathbf{k}_{s}^{\text {scan }}(t)\right)$ was obtained, and re-sampled to shifted time points using the delay determined for the EPI sequence $\left(\tilde{\mathbf{k}}_{s}^{\text {scan }}(t)\right)$. The four $k$-space trajectories were used for image reconstruction of $\mathrm{MR}$ data acquired from the same structured phantom used in the EPI experiments.

For all reconstructions, images from 31 individual elements of a head coil were reconstructed separately using non-uniform Fourier transformations [25], and sampling density compensation [26]. Images from individual coil elements were combined using Sum-of-Squares. In all EPI reconstructions, 7 of 192 samples in the end of each readout were discarded, as $k_{m}^{\mathrm{reg}}(t)$ was not determined for these due to the delay described above, and the temporal gaps in the scanner's sampling when reversing readout gradient polarity. For spiral sequences, the last 4 of 9099 samples were discarded. In generating both $k_{m}^{\mathrm{reg}}(t)$ and $\mathbf{k}_{s}^{\mathrm{reg}}(t), \lambda$ was chosen by 
simple division of the FPGA regularization term by 4 via bit-shifting. Initial testing showed this value to work best among the equally simple alternatives, which are the integer powers of 2 .

\section{Results}

The standard deviation across 50 repetitions of acquiring $k_{m}(t)$ using each of the described approaches and 50 repetitions of acquiring $k_{0}(t)$ (after correction for $\phi_{1}$, but before correction for $\Delta \omega$ ) are depicted in Fig. 1. From the limited relative standard deviation of $k_{m}^{\mathrm{Duyn}}(t)$ (on average $9.94 \cdot 10^{-7}$ ), the time invariance assumption of the system was found valid for the chosen sequence parameters, speaking against this as being contributing significantly to the standard deviation observed for the remaining measures of $k_{m}(t)$. Due to this $k_{m}^{\text {Duyn }}(t)$ and $\mathbf{k}_{s}^{\text {Duyn }}(t)$ were assumed closest to the actual $k_{m}(t)$ and $\mathbf{k}_{s}(t)$, and were therefore used as reference in the following comparisons. The relative standard deviation of the central measurement of $k_{m}(t)$ was used in comparisons of the individual $k$-space trajectory measures, and are summarized in Tab. 2 together with the following findings.

As expected, the relative standard deviation of the non-regularized $k_{m}^{V}(t)$ was found to increase considerably faster than for the other measures of $k_{m}(t)$, and with a non-linear temporal dependency due to the accumulated quantization errors amplified by the inherent integrations. A mix of $t^{1.5}$ and $t^{2}$ dependence is expected from accumulated random noise and residual voltage bias. In comparison, $k_{m}^{I}(t)$ and $k_{m}^{\text {reg }}(t)$ were quantitatively more reproducible, and an approximately linear temporal dependency was observed for their relative standard deviations as ex- 


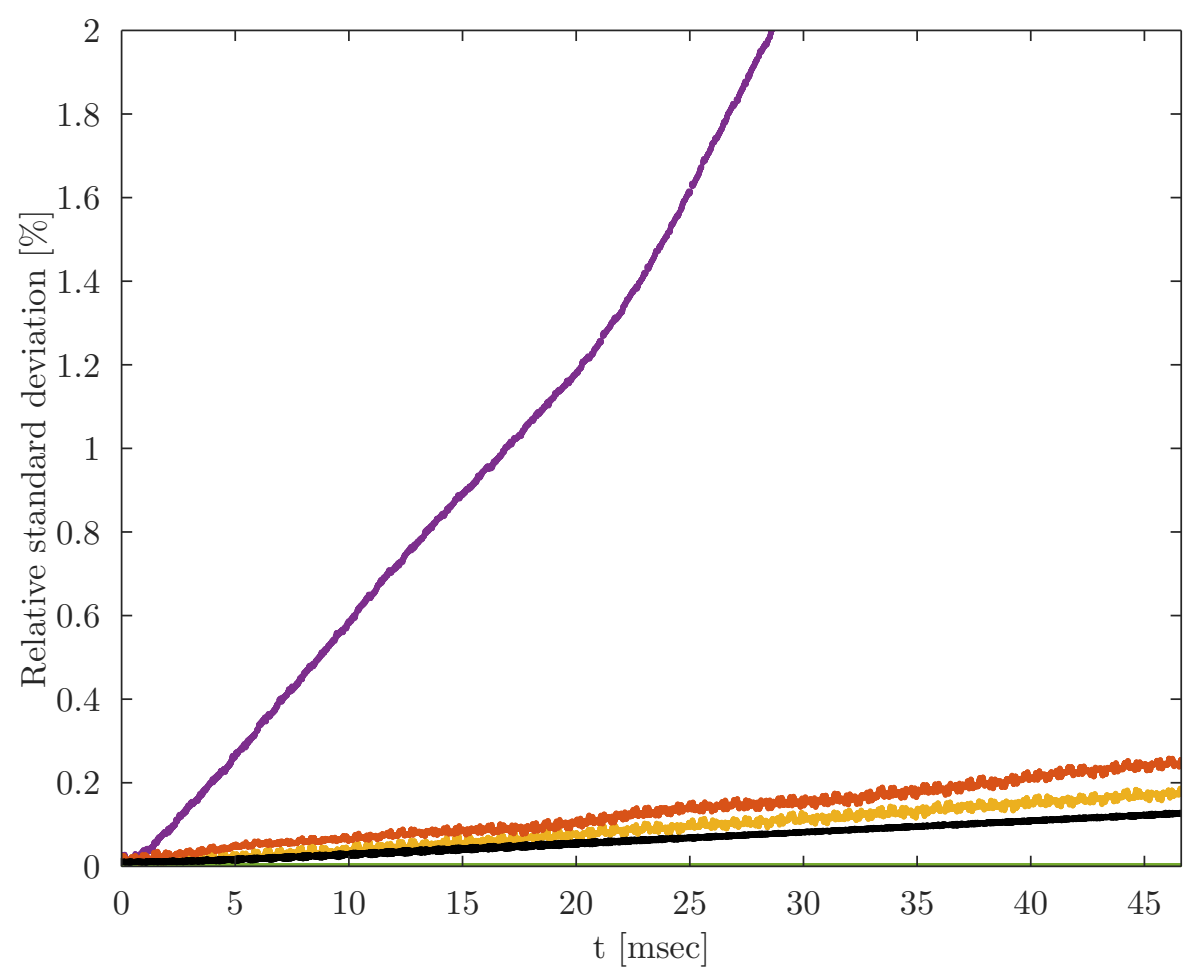

$\square k_{m}^{V}(t) \quad k_{m}^{I}(t) \quad \square k_{m}^{\text {reg }}(t) \quad \square k_{0}(t) \quad \square k_{m}^{\text {Duyn }}(t)$

Fig. 1 Standard deviation in the central $k$-space position across 50 repeated acquisitions of the individual measures of $k_{m}(t)$ and $k_{0}(t)$ relative to the full width of the acquired $k$-space trajectory. The standard deviation of $k_{m}^{V}(t)$ continues with a similar slope after $30 \mathrm{~ms}$, and has a value of $3.99 \%$ after $45 \mathrm{~ms}$.

pected asymptotically for a residual current bias or frequency offset. Notably, the relative standard deviation of $k_{0}(t)$ was comparable to those of $k_{m}^{\text {reg }}(t)$ and $k_{m}^{I}(t)$ (46\% and $67 \%$, respectively, towards the end of readout).

In Fig. 2 and Fig. 3, the time evolution of the average measures of $k_{m}(t)$ and their deviation from $k_{m}^{\text {Duyn }}(t)$ after $\Delta \omega$ and $\phi_{1}$ corrections $(k$-space trajectory er- 
ror) are depicted. In Fig. 3 the top graph depicts the $k$-space trajectory errors for the entire EPI readout, and the bottom graph depicts the 3 central readout periods. All compared $k$-space trajectory errors reached a steady state with oscillations showing periodicity similar to twice the echo-spacing, though with considerably smaller amplitude for $k_{m}^{\text {reg }}(t)$ and $k_{m}^{V}(t)$. In addition to these, approximately $300 \mathrm{~Hz}$ oscillations, diminishing over the first $25 \mathrm{~ms}$, were observed for $k_{m}^{V}(t)$. These oscillations were ascribed to mechanical vibration of the pick-up loop and cabling, and it was confirmed by gentle table-hitting that mechanical resonances exist around this frequency. Disregarding the $300 \mathrm{~Hz}$ oscillations, the averaged $k_{m}^{V}(t)$ showed similar performance to $k_{m}^{\mathrm{reg}}(t)$, but with higher variance between repetitions (not shown), as can also be deduced from Fig. 1.

The $k$-space trajectory error of $k_{m}^{\text {scan }}(t)$ was reduced by $40.2 \%$ from $0.378 \%$ to $0.226 \%$ on average by performing delay correction to yield $\tilde{k}_{m}^{\text {scan }}(t)$. The average $k$ space trajectory error of $k_{m}^{\text {reg }}(t)$ was $0.173 \%$. As evident from Fig. 3 (top), the first readout periods of $k_{m}^{\mathrm{reg}}(t)$ contributed significantly to this error, and disregarding the first six readouts reduced it to $0.076 \%$, (in comparison, the error of $\tilde{k}_{m}^{\text {scan }}(t)$ was $0.213 \%$ for the same period). The cause of this initial inaccuracy was ascribed to the transient error also visible for $k_{m}^{I}(t)$ in Fig. 3, though for $k_{m}^{I}(t)$ the error was only observable for the first two readouts, indicating that the regularization prolonged the time needed to reach steady state.

Using the averaged measures of $k_{m}(t), 2 \mathrm{D}$ images were reconstructed, and scaled so that the reconstruction using $k_{m}^{\text {scan }}(t)$ had an average root-mean-square deviation from the image reconstructed using $k_{m}^{\text {Duyn }}(t)$ (image RMS error) of 1 . Trajectories performing better than the scanner's assumed trajectory will thereby have an RMS error below 1. The spatial distributions of the image RMS error are 


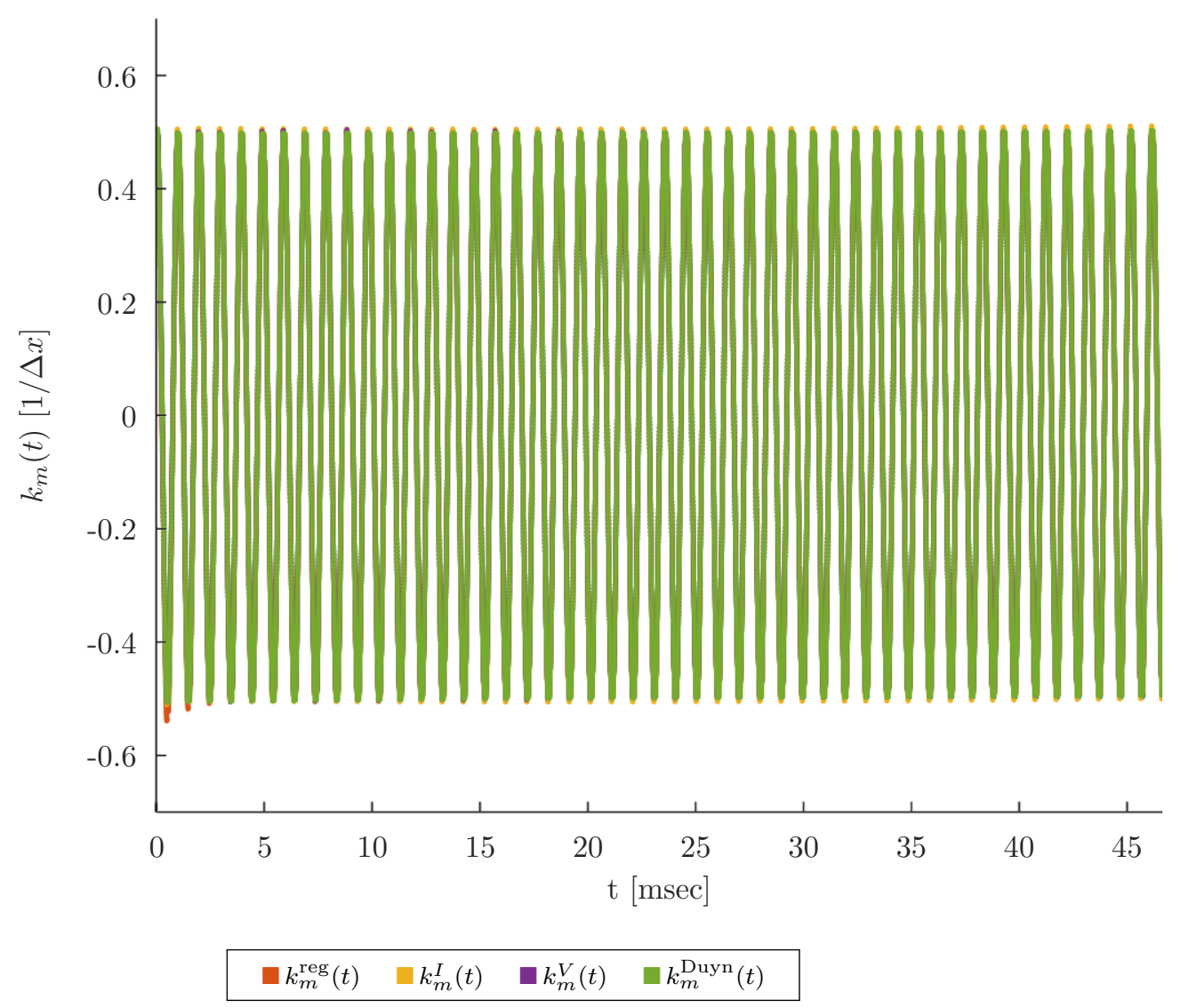

Fig. 2 Averaged measures of $k_{m}(t)$ in units of $1 / \Delta x$, where $\Delta x$ is the voxel size in the reconstructed image in the $\hat{\mathbf{x}}$-direction. The individual measures are almost indistinguishable from $k_{m}^{\text {Duyn }}(t)$ except initially as seen more clearly in Fig. 3.

depicted in Fig. 4 for $k_{m}^{\mathrm{scan}}(t), \tilde{k}_{m}^{\mathrm{scan}}(t), k_{m}^{I}(t)$, and $k_{m}^{\mathrm{reg}}(t)$. The image RMS error of $k_{m}^{V}(t)$ (not shown) had an image RMS error of 0.145 . The image reconstructed using $k_{m}^{\text {scan }}(t)$ showed strong ghosting for the entire phantom, which increased with $\hat{\mathbf{x}}$-distance from the centre of the image. Performing delay correction improved the image significantly, and a similar level of image RMS error was seen for $\tilde{k}_{m}^{\text {scan }}(t)$ 


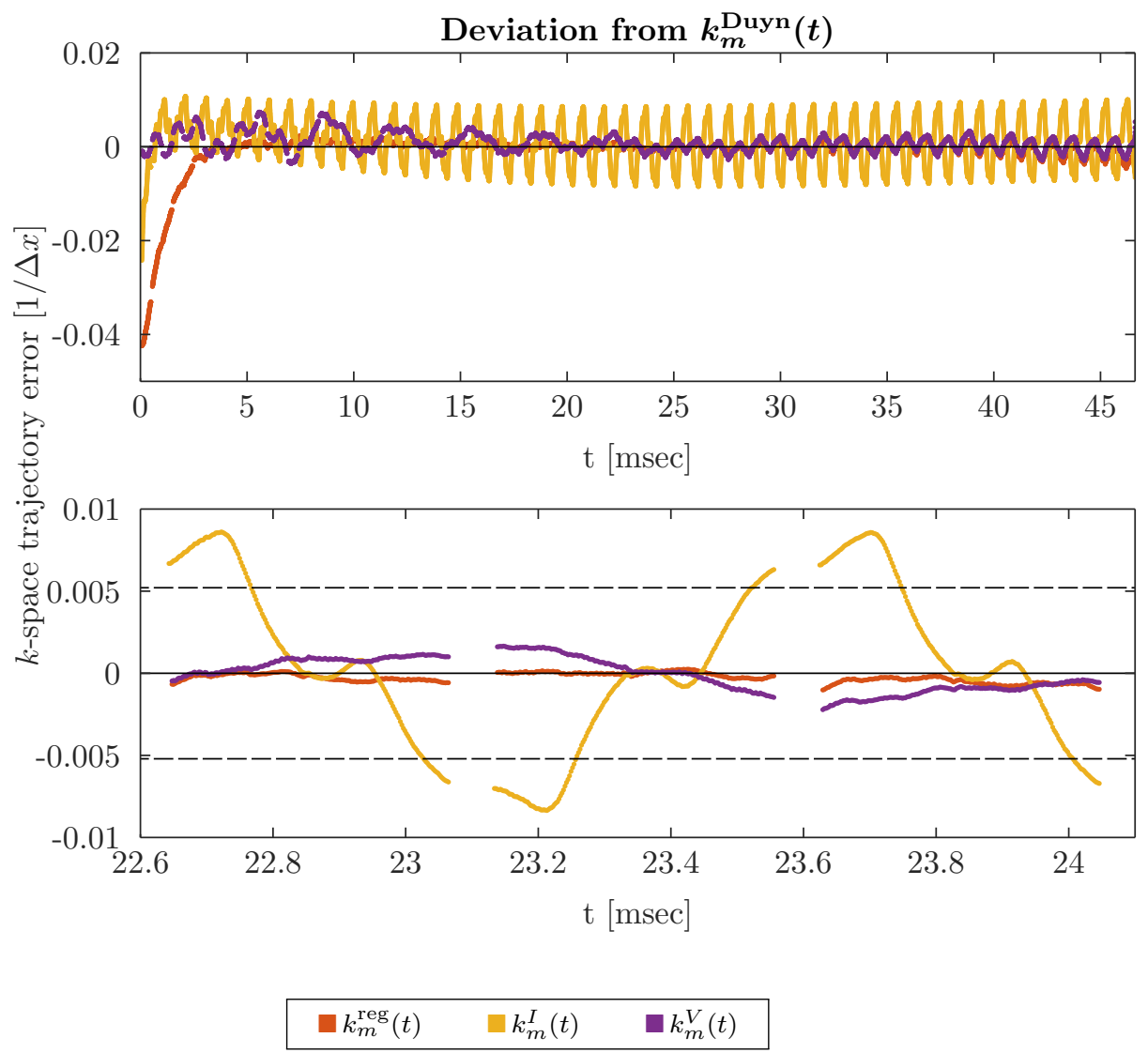

Fig. 3 Difference of acquired measures of $k_{m}(t)$ relative to $k_{m}^{\text {Duyn }}(t)$ (k-space trajectory error) for the entire EPI readout (top), and the centre three readouts (bottom). The dashed lines corresponds to a $k$-space trajectory error of $\pm 0.5 / F O V_{\text {img }}$, with $F O V_{\text {img }}$ being the FOV of the reconstructed images depicted in Fig. 4.

and $k_{m}^{\text {reg }}(t)$. The image RMS error of $\tilde{k}_{m}^{\text {scan }}(t)$ was dominated by contributions from a Nyquist ghost of the edges of the imaged phantom, indicating trajectory errors primarily in outer $k$-space. Ghosting in the image RMS errors of $k_{m}^{\text {reg }}(t)$ was less localised and instead distributed over the phase-encoding direction. The image RMS errors of $k_{m}^{I}(t)$ were similar to those of $\tilde{k}_{m}^{\text {scan }}(t)$ dominated by high-frequency 


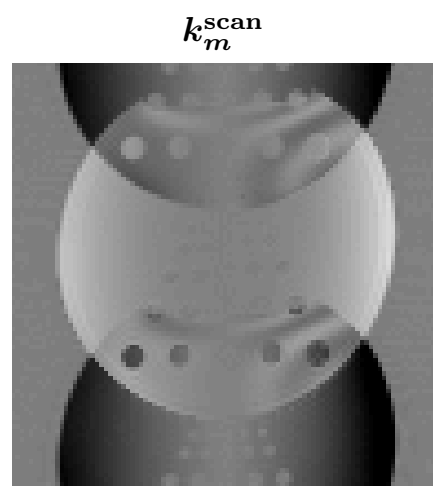

RMS Error: 1

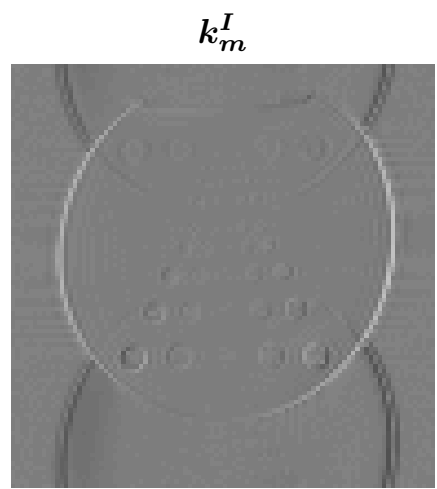

RMS Error: 0.186

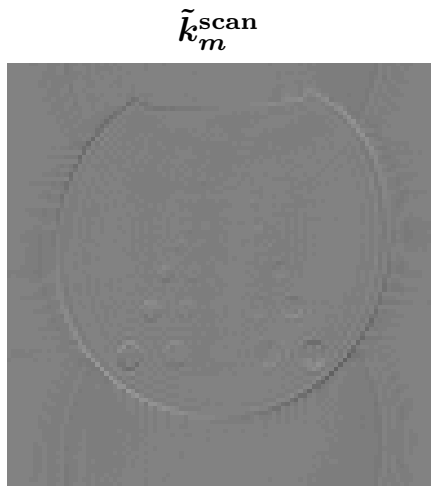

RMS Error: 0.069

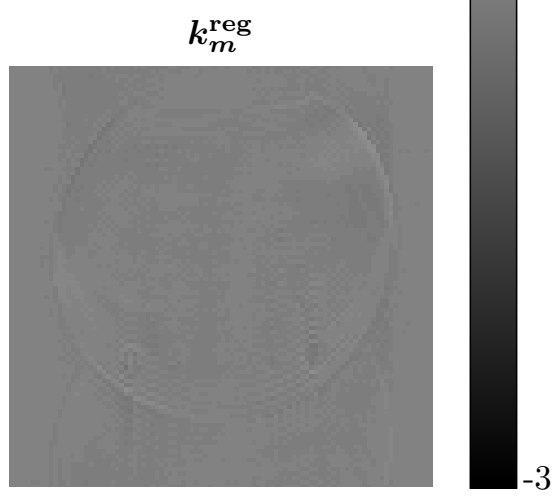

RMS Error: 0.052

Fig. 4 Deviation in reconstructed images compared to the image obtained from reconstruction using $k_{m}^{\text {Duyn }}(t)$ (image RMS error). The nominal phase-encoding gradient was adopted for all reconstructions. The images were normalised to give $k_{m}^{\text {scan }}(t)$ an image RMS error of 1 . The phase-encoding direction, and thereby the direction of ghosting, is up-down.

errors, albeit with higher amplitude. These findings are in concordance with the observations from Fig. 2 and Fig. 3.

The individual components of the spiral $k$-space trajectory are depicted in Fig. 5. Comparable values were observed for $k_{x}(t)$ and $k_{y}(t)$ for each $\mathbf{k}_{s}(t)$-measure. 
At the end of the readout period, the average $k$-space trajectory error of the two 1D-components of $\mathbf{k}_{s}^{\text {scan }}(t)$ was significantly higher than that of $\mathbf{k}_{s}^{\text {reg }}(t)(2.232 \%$ and $0.182 \%$, respectively). The error of $\mathbf{k}_{s}^{\text {scan }}(t)$ was largely caused by a delay of the actual waveform, and $\tilde{\mathbf{k}}_{s}^{\text {scan }}(t)$ showed a $k$-space trajectory error of $0.364 \%$. Reconstructed images from the spiral trajectories are shown in Fig. 6. A rotation of the imaged phantom and inconsistent location of its edges were observed in the image reconstructed using $\mathbf{k}_{s}^{\text {scan }}(t)$ for both $\hat{\mathbf{x}}$ and $\hat{\mathbf{y}}$ directions. Similar was not observed for images reconstructed using $\mathbf{k}_{s}^{\mathrm{Duyn}}(t)$ or $\mathbf{k}_{s}^{\mathrm{reg}}(t)$. While the rotation of the phantom was also not observed in the image reconstructed using $\tilde{\mathbf{k}}_{s}^{\text {scan }}(t)$ (not shown), the artefacts at the border of the phantom persisted, arguing for delay correction not being sufficient for correction of $\mathbf{k}_{s}^{\text {scan }}(t)$. The spatial distributions of the image RMS error are depicted in Fig. 7. While the image RMS errors of all three reconstructions were dominated by contributions close to edges of the phantom, the image RMS error of $\mathbf{k}_{s}^{\text {reg }}(t)$ were $80 \%$ and $76 \%$ lower than those of $\mathbf{k}_{s}^{\text {scan }}(t)$ and $\tilde{\mathbf{k}}_{s}^{\text {scan }}(t)$ respectively.

\section{Discussion}

Using custom circuitry that facilitates sampling of non-MR signals by MR scanners, $k$-space trajectories were encoded into the raw data of imaging sequences. Determining $k$-space trajectories $(k(t))$ solely from digitally integrating an inductively generated signal $(V(t))$ showed limited reproducibility. This was addressed by simple regularization using inaccurate but robust estimates of the gradient from its driving coil current $(I(t))$. The resulting precision and reproducibility of the regularized inductive $k$-space trajectory measure $\left(k^{\mathrm{reg}}(t)\right)$ were compared to those 
Tab. 2 Summary of experimental results relative to $k^{\text {Duyn }}(t)$. The first four columns relate to measures of EPI trajectories, $k_{m}(t)$, and the latter two to spiral trajectories, $\mathbf{k}_{s}(t)$, for which $k^{I}(t)$ and $k^{V}(t)$ were not determined. The standard deviation is given for the centre position of $k$-space.

\begin{tabular}{|c|c|c|c|c|c|c|}
\hline \multirow[b]{2}{*}{ Trajectory } & \multicolumn{4}{|c|}{ EPI } & \multicolumn{2}{|r|}{ Spiral } \\
\hline & Standard dev. & Traj. error & Traj. error (centre) & Image RMS error & Traj. error & Image RMS error \\
\hline$k^{\mathrm{scan}}(t)$ & - & $0.378 \%$ & $0.435 \%$ & 1 & $2.23 \%$ & 1 \\
\hline$\tilde{k}^{\text {scan }}(t)$ & - & $0.226 \%$ & $0.030 \%$ & 0.068 & $0.364 \%$ & 0.856 \\
\hline$k^{\mathrm{reg}}(t)$ & $1.2 \cdot 10^{-3}$ & $0.173 \%$ & $0.032 \%$ & 0.049 & $0.182 \%$ & 0.201 \\
\hline$k^{I}(t)$ & $8.5 \cdot 10^{-4}$ & $0.394 \%$ & $0.058 \%$ & 0.186 & - & - \\
\hline$k^{V}(t)$ & $1.4 \cdot 10^{-2}$ & $0.159 \%$ & $0.079 \%$ & 0.145 & - & - \\
\hline
\end{tabular}

obtained from an exclusively inductive measure $\left(k^{V}(t)\right)$, exclusively from the driving coil current $\left(k^{I}(t)\right)$, the scanner's assumed $k$-space trajectory with and without delay correction $\left(\tilde{k}^{\text {scan }}(t)\right.$ and $k^{\text {scan }}(t)$, respectively), and trajectories determined using Duyn's method $\left(k^{\text {Duyn }}(t)\right)$.

The standard deviation across 50 repetitions was used to evaluate the reproducibility of each of the measures of $k(t)$. Determining $k(t)$ from a digitized inductively generated signal required effectively a double integration, which caused noise to accumulate rapidly. This noise was a primary limitation for the obtainable precision, evident from the standard deviation between repeated acquisitions of $k^{V}(t)$. In comparison, $k^{\text {reg }}(t)$ had significantly lower standard deviation than $k^{V}(t)$, though higher than $k^{I}(t)$. A considerable standard deviation was also ob- 

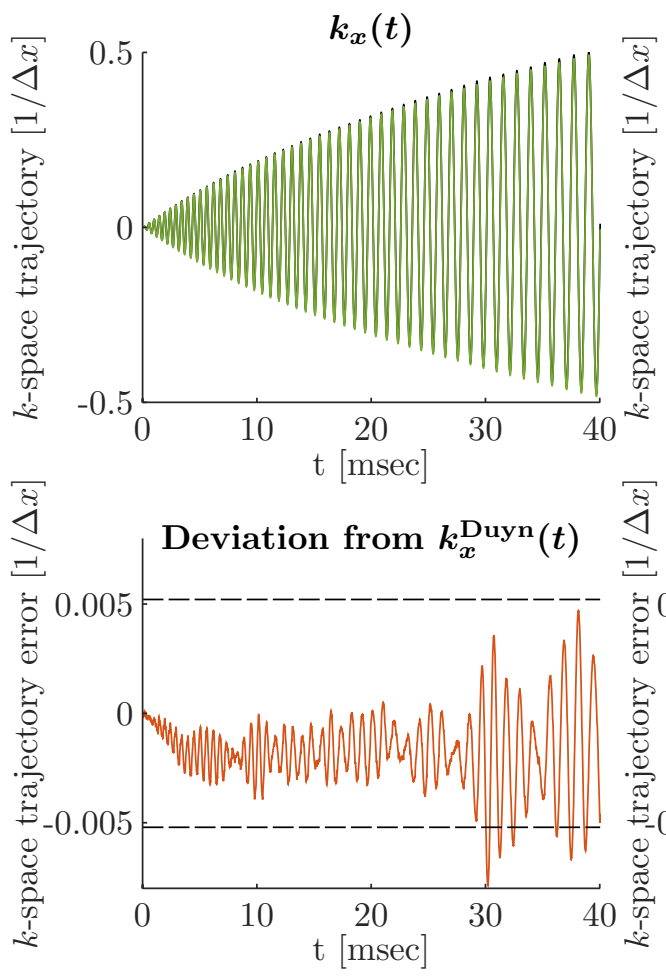

$$
\mathbf{k}_{s}^{\text {reg }}(t) \mathbf{k}_{s}^{\text {Duyn }}(t)
$$

Fig. 5 The individual measures of $k_{x}(t)$ and $k_{y}(t)$ that together yield $\mathbf{k}_{s}(t)$ (top), and the difference to $\mathbf{k}_{s}^{\text {Duyn }}(t)$ (bottom). The trajectories in the 2 top graphs are not easily distinguishable but up to $0.8 \%$ difference from $\mathbf{k}_{s}^{\text {Duyn }}(t)$ is visible in the bottom graphs. The dashed lines corresponds to a $k$-space trajectory error of $\pm 0.5 / F O V_{\mathrm{img}}$, with $F O V_{\mathrm{img}}$ being the FOV of the reconstructed images depicted in Fig. 6 and Fig. 7.

served for acquisitions of a single-frequency signal generated by the circuitry. This argues for a significant contribution to the observed standard deviation resulting from generation of the circuitry-transmitted signal, particularly frequency offsets. 

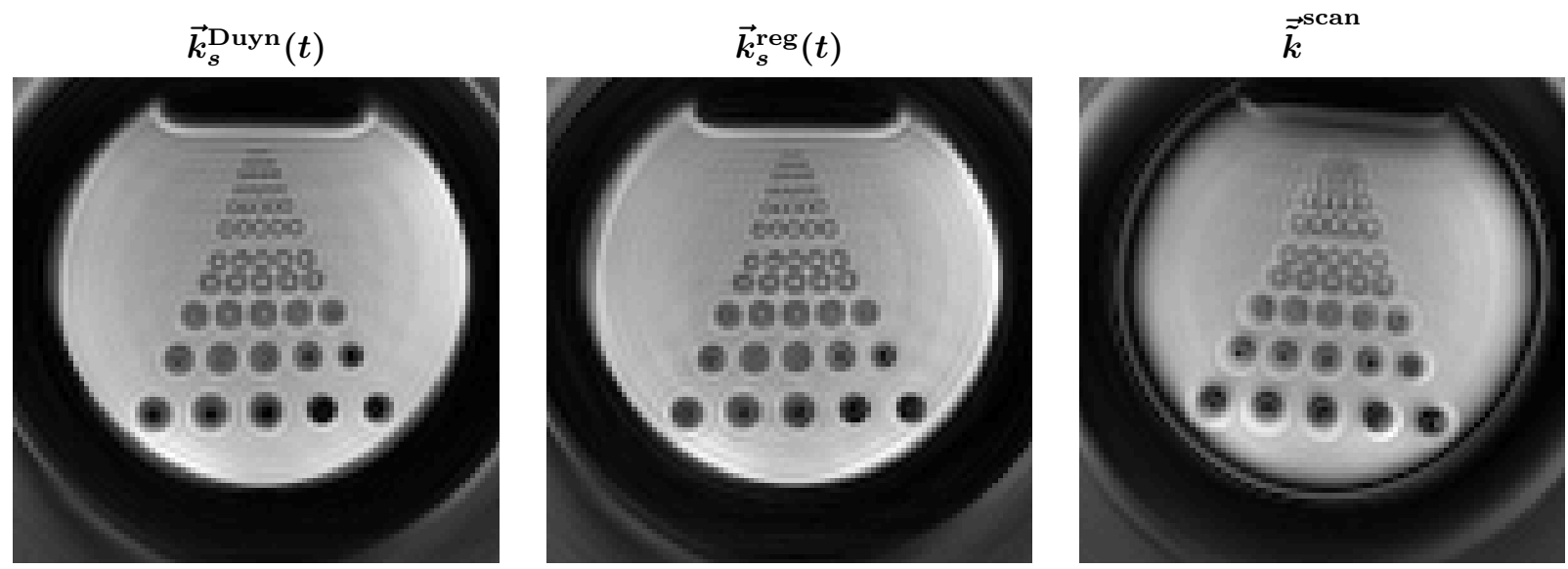

Fig. 6 Reconstructed images using different $k$-space trajectory measures in recon-

struction of MR data acquired during a spiral sequence.

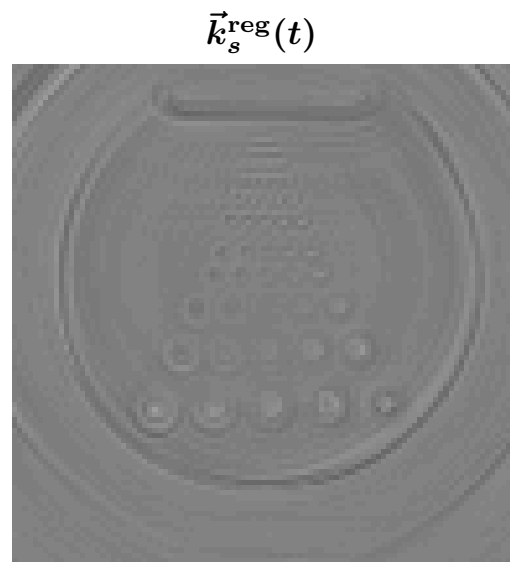

RMS Error: 0.201

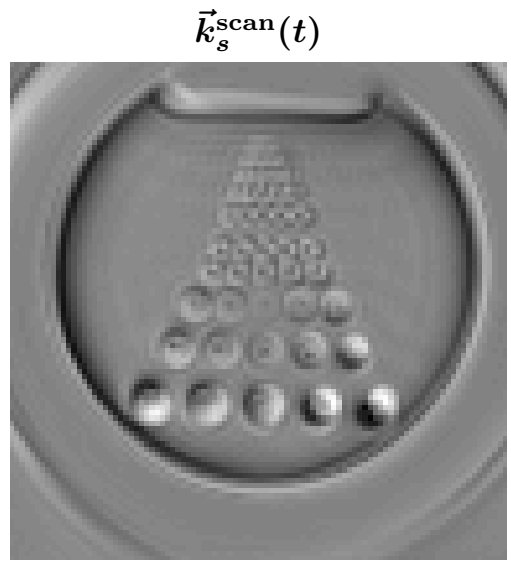

RMS Error: 1

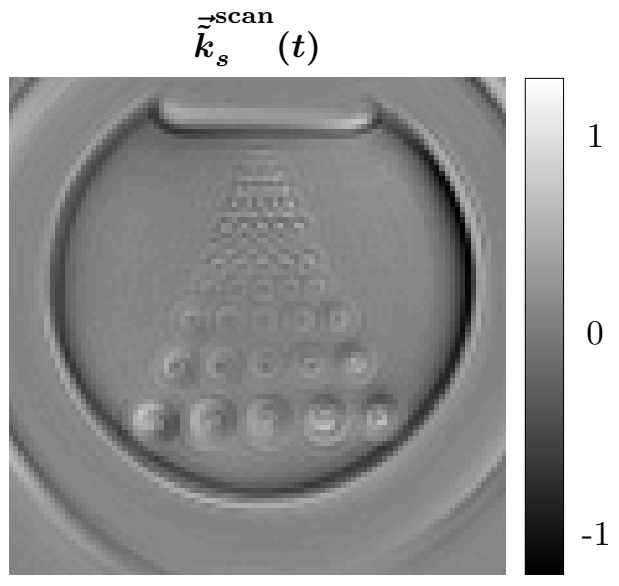

RMS Error: 0.856

Fig. 7 Spatial distribution of the image RMS error for different measures of the $k$-space trajectory of a spiral sequence.

The accuracy of each $k$-space trajectory measure was evaluated by the averaged trajectory's difference from $k^{\text {Duyn }}(t)(k$-space trajectory error) and the RMS difference in reconstructed images (image RMS error). As $k^{I}(t)$ is insensitive to, 
e.g., eddy currents and dependencies of the generated field to the gradient coil's temperature, it is ill-suited to directly predict $k(t)$ on a short time scale, also evident from the observed $k$-space trajectory error and image RMS error of $k^{I}(t)$. A large contributor to the image RMS error of $k^{\text {scan }}(t)$ was an effective delay of the gradient waveforms, and the decrease in image RMS error of $94 \%$ obtained by accounting for an effective delay of $1.4 \mu s$, allude to the level of synchronization necessary to confidently measure $k$-space trajectories. While correction for this delay typically is accounted for in post-processing of EPI sequences, even compared to the corrected trajectory $\tilde{k}^{\text {scan }}(t), k^{\text {reg }}(t)$ showed a smaller $k$-space trajectory error and image RMS error. This was particularly observable for spiral $k$-space trajectories, as $\mathbf{k}_{s}^{\text {reg }}(t)$ showed markedly smaller artefacts than $\tilde{\mathbf{k}}_{s}^{\text {scan }}(t)$. Even though the method clearly improves the eddy current compensation, there is a limit as the sensitivity to slow field changes is inherently low.

In generation of $k^{\text {Duyn }}(t)$, multiple repeated signal acquisitions with varying slice positions were necessary, which caused $k^{\text {Duyn }}(t)$ to benefit from averaging effects reducing its observed standard deviation. This did however not account for the vastly better reproducibility found for $k^{\text {Duyn }}(t)$ compared to the other measured $k$-space trajectories, and Duyn's method is therefore expected to yield higher sensitivity for sequences where SNR is sufficient and where time variation of the gradient system is limited. However, the underlying time-invariance assumption of $k^{\text {Duyn }}(t)$ makes it unsuitable for robust investigation of the accuracy and reproducibility of $k^{\mathrm{reg}}(t)$ acquired concurrently with MR data from gradient intensive sequences. For such investigation, a putative solution is using field probes, as they facilitate concurrent MR and $k$-space trajectory acquisition, and the obtainable sensitivity is similar or superior to Duyn's method [27]. The presented method 
is also expected to be suited for dynamic trajectory estimation. Gradient delays may change with heating but this should not affect the current measure's ability to stabilize the inductive field measure that reflects the dynamic field changes. Ohmic changes of the gradient coils may, however, require updating of the current measure scaling based on the ratio of current and observed field change.

All acquired trajectories have been obtained under assumption of spatially linear gradients, and any non-linearities potentially influence the individual $k$-space trajectory measures differently. An estimate of the linearity can be obtained by comparing differences in the phase evolution from different slice positions when obtaining $k^{\text {Duyn }}(t)$. Here, the largest RMS difference $(2.1 \%$ for $100 \mathrm{~mm}$ difference in slice position) was comparable to what has previously been reported and found to have minimal effect on reconstructed images [20]. The non-linearities are, however, temperature-dependent [3], arguing for the necessity of accounting for these for gradient intensive sequences. Assuming that the non-linearities vary slowly spatially, spherical harmonic functions can be fitted to spatially distributed point-measurements of the field [28, 29], which is feasible with measurements from multiple pick-up coils. Alternatively, the assumption of global linearity can be relaxed to an assumption of local linearity by using gradiometers [15]. It is possible that using a common basis for regularization of the individual measures, such as $I(t)$ or the nominal gradients, limits the obtainable sensitivity, but this remains to be investigated. Similarly, regularization may be improved by, e.g., optimizing $\lambda$ and/or set it to zero in the initial period after excitation where there is no need for regularization and where it causes an initial error in the $k$-space trajectory $k_{m}^{\mathrm{reg}}(t)$. Other algorithms can also be envisioned, but are required to be FPGA-suited. 
Current or field measurements may possibly benefit from using sensors with other noise profiles, or pick-up coils with different sensitivities.

In acquisition of all the acquired $k$-space trajectory measures, the individual spatial components were determined separately, as untangling of multiple contributions was infeasible for single-probe recordings. However, if $V(t)$ is acquired from multiple pick-up coils and $I(t)$ from multiple gradient amplifiers, the individual gradient field components can in principle be disentangled using calibration scans to make the geometrical scaling right for each coil and gradient direction separately, as done here for a single pick-up coil. Since each coil provides a measure of local field changes, and therefore of projections $\mathbf{k} \cdot \mathbf{r}_{i}$ for each coil position $\mathbf{r}_{i}$, three coils are sufficient if placed in different directions from the scanner's isocenter, preferably the physical $\hat{\mathbf{x}}, \hat{\mathbf{y}}$ and $\hat{\mathbf{z}}$ directions. This would facilitate regularization by comparison with an alternative $k$-vector measure derived from three calibrated current measures. If other directions are chosen, the regularization would involve a basis transformation. Given sufficient input channels available (which there are currently not), this additional operation can in principle also be performed in real time using the FPGA featured in the applied circuitry (that notably can be reprogrammed dynamically), at the cost of a prolonged delay of the signal through the circuitry. The additional delay would likely be minimal, as the current delay is dominated by digitization, and updating of the frequency output of the circuitry. The circuitry is limited in the number of external inputs and outputs (4 including $\mathrm{RF}$ and one, respectively), and would require an extension for such simultaneous 3D trajectory mapping ( 7 inputs and 3 outputs, occupying 3 of the scanner's RF channels). 
The method of Duyn et al. [6] relies on encoding a $k$-space trajectory measure into the phase of an MR signal. This makes the obtainable sensitivity directly dependent on the SNR of the MR signal, and thereby challenged by sequences with long readouts, diffusion gradients, or low gamma MRI such as ${ }^{13} \mathrm{C}$ and ${ }^{23} \mathrm{Na}$. The signal may in addition experience dephasing across the excited slice, further limiting the obtainable SNR. The method also relies on correct unwrapping of the phase of the MR signal, effectively limiting the maximum $k$-space position change between sample points. While $k^{\text {reg }}(t)$ likewise is challenged by long readouts due to the accumulation of noise, the obtainable sensitivity is independent of the imaged nucleus, as this simply leads to a change in the frequency of the carrier signal. Also the circuitry allows for free choice of scaling of the ratio between $k$-space trajectory measures and phase of the transmitted signals, making arbitrarily long dwell times and strong gradients manageable.

Only excitatory RF pulses were applied in the performed experiments, and the long TR compared to $T_{1}$ of the imaged phantom ensured that signal contributions from non-primary echoes could be disregarded. The effect of refocusing RF pulses on $k$-space trajectories was therefore not addressed. Determining the tip angle of an RF pulse is not directly feasible from circuitry measurements alone, as only an $\mathrm{RF}$ power-correlate is acquired, yielding only the envelope of applied pulses. One possible approach for handling non-excitatory RF pulses is to assume nominal tip angles, and utilize prior knowledge of distinguishable features, e.g., maximum amplitude, duration or timing to discriminate between pulses. Such prior knowledge can either be coded into memory of the circuitry, or transmitted by the scanner to a receive channel of the circuitry. A further discussion of potential approaches for determining RF pulse characteristics is given in reference [18]. 
In order to obtain synchronization between sampling of $k$-space position and MR data, the generated $k$-space trajectory measure was transmitted via the scanner's receive chain. Other approaches for obtaining synchronization may be envisioned, e.g., direct interfacing to dedicated low-frequency scanner channels for sampling of non-MR signals, or clock-synchronization between scanner and external current/voltage sampling devices. The presented approach was chosen as it allows for an inexpensive, relatively simple, and largely vendor-independent solution, making the measured $k$-space sample points and the MR samples both end up in the scanner's image reconstructor, where they can be used for improving image quality, or even doing more-or-less sequence-agnostic reconstruction. The latter option requires significant further development including RF pulse characterization. The presented approach of combining two measures to estimate actual gradients or $k$-space positions may also be of broader interest, e.g. to vendors or sites with access to scanner subsystems, who can obtain more accurate measures of the gradient current from flux-gates in the amplifiers, for example.

There is considerable potential for further improvement by development of the prototype circuitry implementation here used to demonstrate proof-of-concept. Of the $15.1 \mu \mathrm{s}$ delay through the circuitry, $8 \mu \mathrm{s}$ are due to digitization and communication from the ADCs to the FPGA, and 4.5 $\mu$ s are due to communication from the FPGA to the DDS. The delay can be decreased at the cost of, e.g., reduced ADC linearity, fewer communicated bits, increased expense of the circuitry, or by using parallel communication, which can reduce the delay through the circuitry without affecting the number of bits transmitted. For example, transmission using 4 connections compared to the current single connection would roughly half the total delay through the circuitry. An increased dynamic range or sampling rate of 
the ADC would reduce the quantization error of digitizing $V(t)$ and thereby increase the reproducibility of $k^{V}(t)$. For example, LTC2387-18 (Linear Technology Corporation, Milpitas, CA, USA) facilitates a dynamic range of $120 \mathrm{~dB}$ at $25 \mathrm{kHz}$ sampling rate (with oversampling), $25 \mathrm{~dB}$ more than currently implemented. The price of this ADC would, however, double the total material cost of the circuitry from its current price of 125USD (excluding PCB fabrication), and increase the power consumption of the ADCs 100-fold, making battery-driven usage less attractive. While reducing flexibility in the possible signal processing, an analog mixing of $V(t)$ and $I(t)$ to obtain a measure of $G(t)$ or $k(t)$ may be envisioned, and would reduce the requirements of the ADC due to avoidance of cumulating digitization errors. The limited reproducibility of single-frequency signals generated by the circuitry, indicates that a putative increased performance is achievable with more precise signal-generation than currently available. The performance of the implemented DDS is limited by the frequency of the clock of the circuitry, and up to $30 \mathrm{~dB}$ decrease in DDS-induced noise is achievable by the use of a higher frequency oscillator [30]. This would in turn also decrease the delay through the circuitry, though notably also increase the cost. Implementation of these alternatives and further development can potentially make the circuitry-induced delay insignificant compared to the dwell time of typical MRI acquisitions,

In conclusion, regularized inductive $k$-space trajectory measures were encoded and extracted from signals acquired by an MR scanner. For reconstruction of EPI, the determined $k$-space trajectory performed slightly better than a delaycompensated $k$-space trajectory assumed by the scanner. For spiral trajectories, the regularized inductive measure outperformed the scanner's $k$-space trajectory, and produced images without severe artefacts. The method may offer an inex- 
pensive and relatively simple approach for real-time determination of $k$-space trajectories for development and for improved reconstruction of gradient-demanding sequences. Inductive measures can in principle provide all field components, including non-linearity and concommittant gradients, if they are changing sufficiently fast, but significant theoretical work and extra hardware is needed to go beyond Fourier imaging consistent with recording of a single $k$-space measure (1 to 3 $k$-vector components). While the presented setup only allowed for gradient characterization in one direction at a time, expansion to more dimensions seem relatively straightforward with additional hardware. The method seems generally applicable, also in situations that are potentially challenging for other techniques, e.g., when field inhomogeneities are strong, or when diffusion encoding gradients attenuate MR signals to being near the noise floor.

\section{Acknowledgement}

We acknowledge Dr. Zhentao Zuo for his kind support. We would also like to thank the reviewers for their many good suggestions for improving the paper.

\section{Author contributions}

Study conception and design was performed by all authors. Acquisition of data was performed by Pedersen and L. Hanson using hardware developed by C. Hanson. Analysis and interpretation of data was done by all authors. Drafting of manuscript was done by Pedersen and L. Hanson. Critical revision was done by all authors. 


\section{Compliance with ethical standards}

\section{Conflict of interests}

After completion of the presented work, Pedersen has become an employee of Philips Healthcare.

\section{Ethical approval}

No human subjects or animals were scanned in this study.

\section{References}

1. Barmet C, Zanche ND, and Pruessmann KP. Spatiotemporal magnetic field monitoring for MR. Magnetic Resonance in Medicine, 60(1):187-197, 2008.

2. Dietrich BE, Brunner DO, Wilm BJ, Barmet C, Gross S, Kasper L, Haeberlin M, Schmid T, Vannesjo SJ, and Pruessmann KP. A field camera for MR sequence monitoring and system analysis. Magnetic Resonance in Medicine, 75 (4):1831-1840, 2016.

3. Kasper L, Bollmann S, Vannesjo SJ, Gross S, Haeberlin M, Dietrich BE, and Pruessmann KP. Monitoring, analysis, and correction of magnetic field fluctuations in echo planar imaging time series. Magnetic Resonance in Medicine, 74(2):396-409, 2015.

4. Bruder H, Fischer H, Reinfelder HE, and Schmitt F. Image reconstruction for echo planar imaging with nonequidistant k-space sampling. Magn. Reson. Med., 23(2):311-323, 1992.

5. Delattre BMA, Heidemann RM, Crowe LA, Vallée JP, and Hyacinthe JN. Spiral demystified. Magnetic Resonance Imaging, 28(6):862-881, 2010. 
6. Duyn JH, Yang Y, Frank JA, and van der Veen JW. Simple Correction Method for k-Space Trajectory Deviations in MRI. Journal of Magnetic Resonance, 132 (1):150-153, 1998 .

7. Mason GF, Harshbarger T, Hetherington HP, Zhang Y, Pohost GM, and Twieg DB. A method to measure arbitrary k-space trajectories for rapid MR imaging. Magnetic Resonance in Medicine, 38(3):492-496, 1997.

8. Onodera T, Matsui S, Sekihara K, and Kohno H. A method of measuring fieldgradient modulation shapes. Application to high-speed NMR spectroscopic imaging. Journal of Physics E: Scientific Instruments, 20(4):416-419, 1987.

9. Addy NO, Wu HH, and Nishimura DG. Simple method for MR gradient system characterization and k-space trajectory estimation. Magnetic Resonance in Medicine, 68(1):120-129, 2012.

10. Campbell-Washburn AE, Xue H, Lederman RJ, Faranesh AZ, and Hansen MS. Real-time distortion correction of spiral and echo planar images using the gradient system impulse response function. Magnetic Resonance in Medicine, 75(6):2278-2285, 2016.

11. Vannesjo SJ, Haeberlin M, Kasper L, Pavan M, Wilm BJ, Barmet C, and Pruessmann KP. Gradient system characterization by impulse response measurements with a dynamic field camera. Magnetic Resonance in Medicine, 69(2): 583-593, 2013.

12. Brunner DO, Dietrich BE, Cavusoglu M, Wilm BJ, Schmid T, Gross S, Barmet C, and Pruessmann KP. Concurrent recording of RF pulses and gradient fields - comprehensive field monitoring for MRI. NMR in Biomedicine, 29(9):1162$1172,2016$. 
13. Vannesjo SJ, Dietrich BE, Pavan M, Brunner DO, Wilm BJ, Barmet C, and Pruessmann KP. Field camera measurements of gradient and shim impulse responses using frequency sweeps. Magnetic Resonance in Medicine, 72(2):570583,2014

14. Barmet C, De Zanche N, Wilm BJ, and Pruessmann KP. A transmit/receive system for magnetic field monitoring of in vivo MRI. Magnetic Resonance in Medicine, 62(1):269-276, 2009.

15. Senaj V, Guillot G, and Darrasse L. Inductive measurement of magnetic field gradients for magnetic resonance imaging. Review of Scientific Instruments, 69 (6):2400-2405, 1998.

16. D'Antona G, Lazzaroni M, Ottoboni R, and Svelto C. AC current-to-voltage transducer for industrial application. Instrumentation and Measurement Technology Conference, 2003. IMTC '03. Proceedings of the 20th IEEE, 2(May):11851190 vol.2, 2003.

17. Hanson LG, Lund TE, and Hanson CG. Encoding of electrophysiology and other signals in MR images. Journal of Magnetic Resonance Imaging, 25(5): 1059-1066, 2007.

18. Pedersen JO, Hanson CG, Xue R, and Hanson LG. General purpose electronics for real-time processing and encoding of non-MR data in MR acquisitions. Concepts Magn. Reson. Part B Magn. Reson. Eng., 48(2):e21385, 2018.

19. Encoding of Non-MR signals in scanner recordings, 2018. URL http://drcmr. $\mathrm{dk} /$ MagstripeEncoding.

20. Brodsky EK, Samsonov AA, and Block WF. Characterizing and correcting gradient errors in non-Cartesian imaging: Are gradient errors Linear TimeInvariant (LTI)? Magnetic Resonance in Medicine, 62(6):1466-1476, 2009. 
21. Bernstein MA, King KF, and Zhou XJ. Handbook of MRI Pulse Sequences. 2004. ISBN 9780080533124. doi: 10.1016/B978-0-12-092861-3.X5000-6.

22. Spielman DM and Pauly JM. Spiral imaging on a small-bore system at 4.7t. Magnetic Resonance in Medicine, 34(4):580-585, 1995.

23. Thong YK, Woolfson MS, Crowe JA, Hayes-Gill BR, and Jones DA. Numerical double integration of acceleration measurements in noise. Measurement: Journal of the International Measurement Confederation, 36(1):73-92, 2004.

24. Hiltunen J, Hari R, Jousmäki V, Müller K, Sepponen R, and Joensuu R. Quantification of mechanical vibration during diffusion tensor imaging at $3 \mathrm{~T}$. NeuroImage, 32(1):93-103, 2006.

25. Greengard L and Lee JY. Accelerating the Nonuniform Fast Fourier Transform. SIAM Review, 46(3):443-454, 2004.

26. Pipe JG and Menon P. Sampling density compensation in MRI: Rationale and an iterative numerical solution. Magnetic Resonance in Medicine, 41(1): $179-186,1999$.

27. De Zanche N, Barmet C, Nordmeyer-Massner Ja, and Pruessmann KP. NMR Probes for measuring magnetic fields and field dynamics in MR systems. Magnetic Resonance in Medicine, 60(1):176-186, 2008.

28. Wilm BJ, Barmet C, Pavan M, and Pruessmann KP. Higher order reconstruction for MRI in the presence of spatiotemporal field perturbations. Magnetic Resonance in Medicine, 65(6):1690-1701, 2011.

29. Andersen M, Hanson LG, Madsen KH, Wezel J, Boer V, Van Der Velden T, Van Osch MJ, Klomp D, Webb AG, and Versluis MJ. Measuring motioninduced B0-fluctuations in the brain using field probes. Magnetic Resonance in Medicine, 2016. 
30. Analog Devices; Inc. AD9951 direct digital synthesize (data sheet, Rev. B), 2016. 